\title{
Electromyographic study of shoulder and acromioclavicular joint muscles in women who underwent unilateral breast surgery of the types mastectomy and quadrantectomy
}

\author{
Antonia Dalla Pria Bankoff ${ }^{1,2}$, Sonia Regina Jurado ${ }^{2}$ \\ ${ }^{1}$ Postural Assessment Laboratory, FEF-Unicamp, Campinas, Brazil; dallabankoff@bol.com.br \\ ${ }^{2}$ Federal University of Mato Grosso do Sul-Campus de Três Lagoas, Três Lagoas, Brazil
}

Received 10 September 2013; revised 15 October 2013; accepted 26 October 2013

Copyright (c) 2013 Antonia Dalla Pria Bankoff, Sonia Regina Jurado. This is an open access article distributed under the Creative Commons Attribution License, which permits unrestricted use, distribution, and reproduction in any medium, provided the original work is properly cited.

\section{ABSTRACT}

We studied 20 women with mean age 57.7 years, being 9 with unilateral quadrantectomy surgery, 1 with surgery type bilateral quadrantectomy and 10 with unilateral mastectomy surgery. The average operative time was 9.8 (nine years and eight months). We studied using surface electromyography the mean deltoid, upper trapezius and latissimus dorsi muscles in order to check the action potentials of these muscles when performing a sequence of movements of these joints. It was used for the study, an Acquisition Data System ADS1000 containing 12 channels. The electromyography (EMG) results expressed in RMS (Root Mean Square) were analyzed and compared between the surgical and nonsurgical side, among the three repetitions of the sequence of movements and between mastectomy and quadrantectomy for each muscle. For statistical analysis we used analysis of variance (ANOVA) with a double repetition factor $(p<0.05)$ for the EMG results. The results showed a significant difference $(p<0.05)$ between the surgical and non-surgical sides to the muscles studied. There was no significant difference between the three repetitions of sequential movements in electromyographic analysis to the muscles studied. There was no significant difference between the types of surgery (mastectomy and quadrantectomy) for the muscles studied.

Keywords: Electromyography; Skeletal Muscle;
Breast Surgery; Quadrantectomy; Mastectomy

\section{INTRODUCTION}

The breasts are glands and their main function is to produce milk. They are composed of lobes that divide into smaller portions; the lobules and ducts that carry milk produced outwards through the nipple. Like all other organs of the human body, they are also found in breast blood vessels that supply blood to the breast, and lymphatic vessels through which lymph flows. Lymph is a clear liquid that has a function similar to the blood to carry nutrients to various parts of the body and collect undesirable substances. The lymphatic vessels are grouped in what is called lymph nodes, or swollen lymph nodes. The lymphatic vessels drain into the breast glands in the armpits (lower arms) in the neck and chest [1].

Breast cancer occurs when cells of the body begin to divide and reproduce very quickly and haphazardly. Most breast cancer affects the cells of the ducts of the breasts. Therefore, the most common breast cancer is called ductal carcinoma. It can be in situ, when there is nothing of the first cell layers of duct or attacker, when invading the surrounding tissue. Cancers that begin in the breast lobules are called lobular carcinoma and are less common than the first. This type of cancer most often affects both breasts. Carcinoma Inflammatory breast cancer is rarer and usually presents itself aggressively, compromising the entire breast, leaving it red, swollen and hot [1].

As a result of breast cancer treatment, several complications have been reported, among them, including those resulted from surgery. The surgical techniques can be 
used for the mastectomy or conservative surgery, which may be associated with axillary lymphadenectomy (AL) or sentinel lymph node biopsy [2]. Among the surgical complications of AL, it is the long thoracic nerve injury [3]. Most injuries of this nerve are partial and transient (neuropraxia), which gradually recover with conservative treatment [2]. However, when this regression does not occur in the first six months, the lesion can be considered complete, with few chances of rehabilitation [4].

The long thoracic nerve injury results in decreased strength or paralysis of the serratus anterior muscle, leading to destabilization of the shoulder girdle on the prominence of the medial border of the scapula and inferior angle of rotation of the middle line, featuring a winged scapula [5]. In addition to the specific lesion of the long thoracic nerve, muscle changes are also seen as morbidity related to the muscles in the shoulder joint and scapular stabilization and, we can cite, in addition to the serratus anterior muscle, others such as the upper trapezius and deltoid medium [6].

After the AL in the treatment of breast cancer, the prevalence of scapular winging has varied 1.5\% - 12.6\% [7] and the incidence of $8 \%$ to $30 \%$ [8]. In a cross-sectional study conducted in Rio de Janeiro, the prevalence of $6.3 \%$ in women with seven to 287 months elapsed after the AL was observed. In an incidence study with 599 women who underwent AL, we observed the presence of winged scapula in $69.5 \%$ of patients evaluated in the immediate postoperative period [9].

The efficacy of physiotherapists, as well as the recovery of normal movement in cases of neuropraxia may be accompanied by physical examination. However, it is believed that the use of objective methods such as surface electromyography, for example, will provide more reliable results on the evolution of the clinical status of patients [10]. The EMG is defined as a non-invasive experimental technique, which consists of the development, registration and analysis of myoelectric signals [11]. The myoelectric activity through the electromyographic signal, based on the action potential of the plasma membrane of muscle fibers, results from the process of depolarization and repolarization [4]. The root mean square electromyographic signal (RMS) is one of the parameters used to measure muscle electrical activity and indicates changes in firing frequency and range of muscle activity, so that the higher the value-and, consequently, the range is greater - the greater the muscle electrical activity [12].

\section{Decreased Range of Shoulder Motion}

Range of motion (ROM) is the complete movement possible of a segment being maintained by periodic movement of the member [13]. Some factors may lead to impairment of ROM. Among them we emphasize the surgical aggression and inactivity or immobilization of the limb. In case of realization of the radical mastectomy, the pectoralis major and minor are removed, resulting in a decrease in strength and upper limb function involved. The same occurs when the nerve of Bell is temporarily traumatized during axillary dissection, resulting in weakness of the serratus anterior muscle, disrupting the movement of the scapula and shoulder abduction on the affected side $[14,15]$. Re-education of upper limb girdle is a basic need in the patient undergoing surgery for breast cancer, whatever the surgical technique is. Its main objective is to restore limb function as soon as possible [16].

\section{MATERIALS AND METHODS}

We studied the middle deltoid, upper trapezius and latissimus dorsi by surface electromyography in order to check the action potentials of these muscles in women affected by breast surgeries types unilateral mastectomy and quadrantectomy

\section{POPULATION}

We studied 20 women with a mean age 57.7 years; being 9 with unilateral quadrantectomy surgery, 1 with bilateral quadrantectomy surgery and 10 with unilateral radical mastectomy surgery. The average operative time was 9.8 (nine years and eight months). The electromyographic study was conducted with women who underwent this surgery in a period longer than six months of surgeries and that performed all sessions of physical therapy after the surgery recommended by the physician and the SUS after surgery being a total of 12 sessions.

\section{Method}

\subsection{Instruments}

It was used in the study, a data acquisition system ADS1000 12-channel of analog input for simple signs, $50 \times$ gain, $1-1000 \mathrm{~Hz}$ band (1), 12 differential analog input channels, gains $1 \times, 100 \times, 300 \times$ and $600 \times$ selectable in factory, 0 - $1000 \mathrm{~Hz}$ band (2), high-pass filter with cutoff frequencies selectable in software options: 1 $\mathrm{Hz}, 2 \mathrm{~Hz}, 5 \mathrm{~Hz}, 10 \mathrm{~Hz}, 20 \mathrm{~Hz}, 50 \mathrm{~Hz}$ or without filter; Low Pass Filter with upper cutoff frequencies selectable software for the options: $100 \mathrm{~Hz}, 200 \mathrm{~Hz}, 300 \mathrm{~Hz}, 500$ $\mathrm{Hz}, 1000 \mathrm{~Hz}$ or without filter; Filters "Notch" operated by software in the options: $60 \mathrm{~Hz}, 120 \mathrm{~Hz}, 180 \mathrm{~Hz}$ and $240 \mathrm{~Hz}$ and 2 optically isolated digital inputs, 2 digital outputs optically isolated acquired by FAPESP Process No. 2010/08923-2.

\subsection{Procedures and Data Acquisition}

All research subjects; dressed only a brassiere, initially, 
it was performed the posterior superior asepsis of the thorax and upper arm, thus preparing the sites for electrode placement, avoiding any interference in the acquisition of electromyographic signals.

Surface electrodes with electric-conductive gel were fixed on the skin in the respective muscles. For the latissimus dorsi muscle in the lower back and side of the chest, deltoid medium in the ventral portion of the upper trapezius. We analyzed the muscle action potentials bilaterally, regardless of the side where the surgery took place (right or left). Each research subject underwent 3 proposed sequences of moves.

The study was conducted at the Laboratory of Electromyography and Biomechanics of Posture, School of Physical Education-Unicamp. For each procedure before starting data acquisition, the subject was asked three times to conduct the activities of sequential movements of the shoulder joint, thus avoiding some sort of error during execution.

All subjects in the study signed an informed consent for participation in the research. The project was approved by the Ethics Committee of Unicamp under No. 984/2010.

\section{MOVEMENTS REVIEWED}

Hyper-flexion and extension of the arm, arm abduction and adduction, extension and flexion of the arm horizontal, internal and external rotation of the arm, lifting and lowering the shoulder.

\section{RESULTS}

In our work we studied the electrical potential of muscle cells in skeletal muscles acting on the shoulder joint of women who underwent breast surgeries due to breast cancer. When women perform these types of surgeries (mastectomy and quandrantectomia) are removed completely or partially muscles around the shoulder joint, for example muscles pectoralis major and minor and other muscles. Then, using electromyography to measure the action potentials of the latissimus dorsi, upper trapezius and middle deltoid in women who have these types of surgeries. What is electromyography? When we speak of electromyography signals, we talk about "Generation of Action Potential". Therefore, an action potential is an electrical control type all or nothing produced by the cell in response to the impulse received. The signals are action potentials generated by an electric control between neurons and muscle fibers. This means both neurons as muscle fibers can generate action potentials, in other words, the impulses received by the neuron, which are the basis for the generation of the action potential. In this sense, it is much easier to understand the relationship between the action potential and electromyography signals.
The trapezius muscle shown in Figure 1 had the lowest average RMS values of EMG signals on the type of mastectomy surgery, although the figures presented by the type quadrantectomy hand surgery were not as impressive. When we look at this graph the surgical side considering both types of surgery, we found that the values are lower. With the results, it is quite evident that both types of surgery compromise the kinesiology of the trapezius muscle in relation to the joints studied. One of the complaints of women is the difficulty of raising the shoulder of the hand surgery to perform daily tasks and also complain that the non-surgical side does not offer force necessary to accomplish necessary task on a daily basis.

Figure 2 shows the results of latissimus dorsi muscle. One can check, on the latter, that the lowest RMS values through the acquisition of electromyography signals are

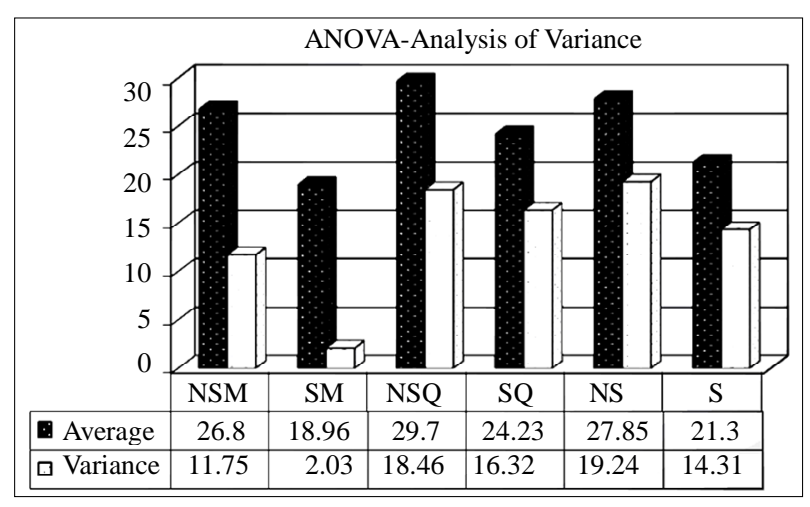

Figure 1. Income statement in RMS (Root Mean Square) on the electromyography evaluation in women mastectomized and quadrantectomized on the Trapezius muscle. Non-Surgical Mastectomy (NSM), Surgical Mastectomy (SM), Non-Surgical Quadrantectomy (NSQ), Surgical Quadrantectomy (SQ), Non-Surgical (NS) and Surgical (S).

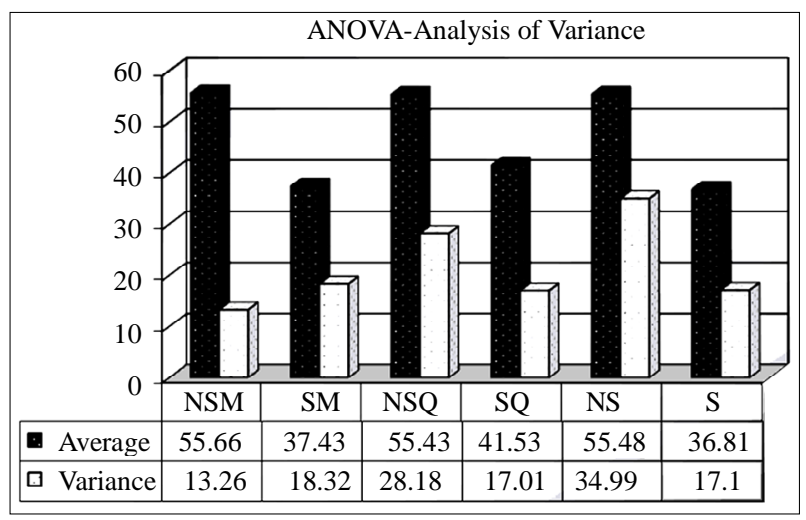

Figure 2. Income statement in RMS (Root Mean Square) for electromyography evaluation in women mastectomized and quadrantectomized on the latissimus dorsi muscle. Non-Surgical Mastectomy (NSM), Surgical Mastectomy (SM), Non-Surgical Quadrantectomy (NSQ), Surgical Quadrantectomy (SQ), Non-Surgical (NS)and Surgical (S). 
scored on the type of surgery and hand surgery Mastectomy both types of surgery. The results presented aimed to be a muscle that suffer the consequences in their kinesiology types of breast surgeries. It seemed to us to be more engaged in radical mastectomy surgery, but in the quadrantectomy surgery was also weakened in relation to the achievement of the sequence of movements for the shoulder joint. In the adduction movement women demonstrated great difficulty in approaching the body side arm with surgery. It is the primary motor adduction, extension and hyperextension of the shoulder joint, helping also in the horizontal extension and internal rotation. It is a muscle that works in many movements of the shoulder joint and also interferes with the body posture taking into account its origin from the ilium, through the lumbar, thoracic, and its insertion to the humerus at the back. As a result, it may change the biomechanical relationship between shoulder and pelvis, causing misalignment between the sacrum and the ilium bones bilaterally, asymmetrically tilting the pelvis, or pulling your shoulders down causing drooping shoulders. For women with mastectomy and quadrantectomy surgery the inability can cause postural problems [17].

Figure 3 shows the results of the Middle Deltoid muscle, which showed low values in RMS through the acquisition of electromyography signals. Considering its kinesiology as a whole performs the abduction of the arm, specifically the middle portion. Part clavicular and acromial portions of adjacent flex the arm, the spinal and adjacent parts of the acromial portions extend arm. The ventral portion rotates the arm medially and laterally dorsal portion.

Considering the kinesics trapezius muscle rotates the scapula, raising the acromial extremity in abduction and full flexion of the arm. The top acting alone elevates the

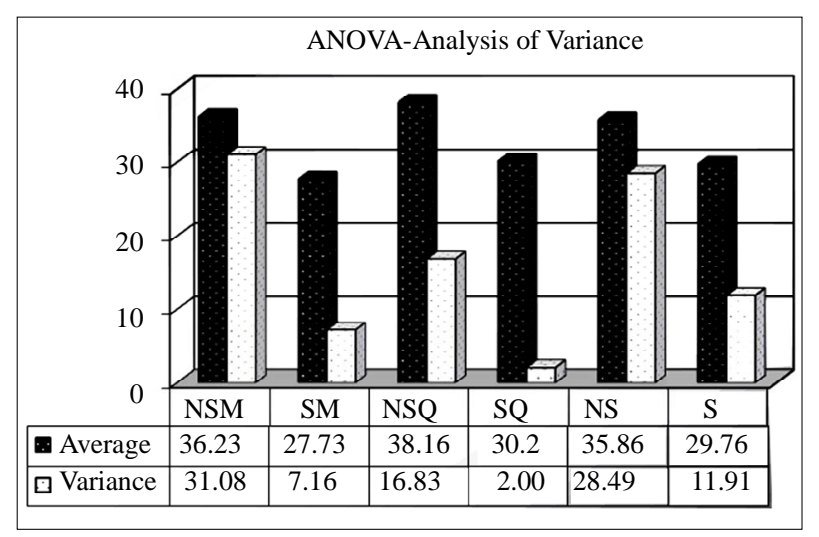

Figure 3. Statement of the results in RMS (Root Mean Square) on the electromyography evaluation in women mastectomized and quadrantectomized on the Middle Deltoid muscle. NonSurgical Mastectomy (NSM), Surgical Mastectomy (SM), NonSurgical Quadrantectomy (NSQ), Surgical Quadrantectomy (SQ), Non-Surgical (NS)and Surgical (S). scapula cranially, elevating the shoulder, then it has an important participation from the shoulder joint. It is important to note that when all portions of the trapezius contract simultaneously, they act more on the upper portion than on the lower portion of the scapula. It should be noted the effect of posture, the action of the trapezius muscle. When the shoulder is the most high, or when a weight is supported on the shoulders, with the individual standing, the second portion is contracted vigorously and, third, little or nothing [17].

One of the difficulties of women with breast surgeries types of mastectomy and quadrantectomy is the realizetion of abduction movement of the arm because the muscles that control the movements of the shoulder joint to be affected by surgery. It showed electromyographic signal after 45 degrees of abduction of the arm, stopping soon after, because most could not reach the 90 degrees of amplitude. If we look in depth at Figure 3 we see that the values shown for the mastectomy surgery side, quadrantectomy surgery side and surgical side with both types of surgery, the difference found between the values is very small. This leads us to believe that the middle deltoid muscle kinesiology suffer consequences with both types of surgery.

\section{STATISTICAL RESULTS ABSTRACT}

For statistical analysis we used analysis of variance (ANOVA) with a double repetition factor $(\mathrm{p}<0.05)$ for the EMG results. The results showed significant difference ( $p<0.05$ ) between the surgical and nonsurgical sides for all muscles studied. There was no significant variation between the three sets of repetitions for all muscles studied. There was no significant difference between the types of surgery (mastectomy and quadrantectomy) for the trapezius, latissimus dorsi and Middle Deltoid.

\section{DISCUSSION}

When I discussed the project and drafted the literature impressing me, the placements of some authors say that the recovery was long overdue. The long thoracic nerve injury results in decreased strength or paralysis of the serratus anterior muscle, leading to destabilization of the shoulder girdle on the prominence of the medial border of the scapula and inferior angle of rotation of the middle line, featuring a winged scapula [5]. [6] describes that in addition to the specific lesion of the long thoracic nerve, muscle changes are also seen as morbidity related to the muscles in the shoulder joint and scapular stabilization and can cite, in addition to the serratus anterior muscle, others such as the upper trapezius and middle deltoid [4]. Reports that when this regression does not occur in the first six months, the lesion can be considered complete, 
with few chances of rehabilitation.

Considering the kinesiology of the muscles studied in relation to the shoulder joint and acromioclavicular which act in many of the movements of these joints, it was evident that the types of mastectomy and quadrantectomy surgeries impair the quality of surgical hand movements specifically, however, the non-surgical side also suffers from some kind of commitment in the performance of movements and also in relation to body posture due to overload $[1,18]$.

In our results electromyographic and Middle Deltoid muscle Trapezius had lower RMS values representing the action potentials of muscles in the sequence of movements performed, specifically the surgical side confirming the findings of [6]. An observation, however, not assessed by this project is that these types of surgeries and more the problem of the disease also affect the next non-surgical, body posture, body balance, muscle strength and more emotional, mainly because they feel fear and pain on movement. They complain much of the limitations on range of motion of these joints, especially for performing everyday tasks.

\section{CONCLUSIONS}

Based on the results we believe we can draw the following conclusions regarding the three parts of our experimental work:

- There was a significant difference between surgical and nonsurgical sides ( $<$ 0.05) for the muscles studied;

- There was no significant difference between the three replicates during analysis of electromyography to the muscles studied;

- There was no significant difference between the types of surgery (mastectomy and quadrantectomy) ( $\mathrm{p}<$ 0.05 ) for the muscles studied.

\section{REFERENCES}

[1] Bankoff, A.D.P. (2012) Electromyographic study of shoulder and acromioclavicular joint muscles in women who underwent unilateral breast surgery of the types mastectomy and quadrantectomy. Sport Inspiring a Learning Legacy, Routledge Online Studies, Glasgow, 2, 79142.

[2] Langer, I., Guller, U., Berclaz, G., Koechli, O.R., Schaer, G., Fehr, M.K., et al. (2007) Morbidity of sentinel lymph node biopsy (SLN) alone versus SLN and completion axillary lymph node dissection after breast cancer surgery: A prospective Swiss multicenter study on 659 patients. Annals of Surgery, 245, 452-461. http://dx.doi.org/10.1097/01.sla.0000245472.47748.ec

[3] Freitas Júnior, R., Ribeiro, L.F.J., Taia, L., Kajita, D., Fernandes, M.V. and Quiroz, G.S. (2001) Linfedema em pacientes submetidas à mastectomia radical modificada. Revista Brasileira de Ginecologia e Obstetrícia, 23, 205-
208.

http://dx.doi.org/10.1590/S0100-72032001000400002

[4] Campbell, W.W. (2008) Evaluation and management of peripheral nerve injury. Clinical Neurophysiology, 119, 1951-1965. http://dx.doi.org/10.1016/j.clinph.2008.03.018

[5] Schmitz, C., Sodian, R., Witt, T.N., Juchem, G., Lang, N., Bruegger, C., et al. (2009) Winged scapula after aortic valve replacement. The Annals of Thoracic Surgery, 87, 1277-1279. http://dx.doi.org/10.1016/j.athoracsur.2008.08.050

[6] Shamley, D.R., Srinanaganathan, R., Weatherall, R., Oskrochi, R., Watson, M., Ostlere, S., et al. (2007) Changes in shoulder muscle size and activity following treatment for breast cancer. Breast Cancer Research and Treatment, 106, 19-27. http://dx.doi.org/10.1007/s10549-006-9466-7

[7] Sahin, F., Yilmaz, F., Esit, N., Aysal, F. and Kuran, B. (2007) Compressive neurophaty of long thoracic nerve and accessory nerve secondary to heavy load bearing. A case report. Europa Medicophysica, 43, 71-74.

[8] Paim, C.R., de Paula Lima, E.D., Fu, M.R., de Paula Lima, A. and Cassali, G.D. (2008) Post lymphadenectomy complications and quality of life among breast cancer patients in Brazil. Cancer Nursing, 31, 302-309. http://dx.doi.org/10.1097/01.NCC.0000305747.49205.b1

[9] Bergmann, A., Mattos, I.E., Koifman, R.J., Ribeiro, M.J. P., Nogueira, E.A., Oliveira, A.C.G., et al. (2005) Incidência de complicações no pós-operatório imediato: Análise descritiva de 599 mulheres submetidas a linfadenectomia axilar no câncer de mama. Congresso Brasileiro de Mastologia, Rio de Janeiro, 146-329.

[10] Wolf, S.L., Butler, A.J., Alberts, J.L. and Kim, M.W. (2005) Contemporary linkages between EMG, kinetics and stroke rehabilitation. Journal of Electromyography \& Kinesiology, 15, 229-239. http://dx.doi.org/10.1016/j.jelekin.2005.01.002

[11] Hermens, H.J., Freriks, B., Disselhorst-Klug, C. and Rau, G. (2000) Development of recommendations for SEMG sensors and sensor placement procedures. Journal of Electromyography \& Kinesiology, 10, 361-374. http://dx.doi.org/10.1016/S1050-6411(00)00027-4

[12] Goodman, B.E. (2008) Channels active in the excitability of nerves and skeletal muscles across the neuromuscular junction: Basic function and pathophysiology. Advances in Physiology Education, 32, 127-135. http://dx.doi.org/10.1152/advan.00091.2007

[13] Kisner, C. and Colby, L.A. (1998) Exercícios terapêuticos: Fundamentos e técnicas. Manole, São Paulo, 56-95.

[14] Box, R.C., Reul-Hirche, H.M., Bullock-Saxton, J.E. and Furnival, C.M. (2002) Physiotherapy after breast cancer surgery: Results of a randomized controlled study to minimiselymphoedema. Breast Cancer Research and Treatment, 75, 51-64. http://dx.doi.org/10.1023/A:1016591121762

[15] Camargo, M.C. and Marx, A.G. (2000) Reabilitação física no câncer de mama. Editora Roca, São Paulo, 79-93.

[16] Nagel, P.H., Bruggink, E.D., Wobbes, T. and Strobbe, L.J. (2003) Arm morbidity after complete axillary lymph node dissection for breast cancer. Acta Chirurgica Belgica, 103, 
212-216.

[17] Bankoff, A.D.P. (2007) Morfologia e cinesiologia aplicada ao movimento humano. Editora Guanabara Koogan, Rio de Janeiro, 143-157.

[18] Seghers, J. and Spaepen, A. (2004) Muscle fatigue of the elbow flexor muscles during two intermittent exercise protocols with equal mean muscle loading. Clinical Biomechanics, 19, 24-30.

http://dx.doi.org/10.1016/j.clinbiomech.2003.08.003 